\title{
Effect of Channel Estimation Error on Bit Rate Performance of Time Domain Equalizers
}

\author{
Ming Ding*, Brian L. Evans**, and Ian Wong** \\ *Bandspeed, Inc. 4301 Westbank Drive, Austin, TX 78746 \\ **Dept. of Electrical and Computer Eng., The University of Texas, Austin, TX 78712 \\ E-mail: mding@bandspeed.com, \{bevans, iwong\}@ece.utexas.edu
}

\begin{abstract}
Channel equalization plays a key role in achieving high bit rates in wireline multicarrier systems. Some VDSL systems and all standardized multicarrier ADSL systems employ time domain equalization (channel shortening) and frequency domain equalization in channel equalization. In this paper, we analyze the impact of imperfect channel estimates on the bit rate performance of four time domain equalization methods. We derive a closed-form expression for the bit rate loss due to channel estimation error. We simulate the sensitivity in bit rate performance using firstgeneration downstream ADSL transmission. In simulation, the minimum intersymbol interference and minimum mean square error methods are relatively insensitive to channel estimation errors vs. minimum delay spread and maximum shortening signal-to-noise ratio methods.
\end{abstract}

\section{INTRODUCTION}

In the late 1990s, standardization of ADSL and cable modems enabled widespread deployment of high-speed data communications to home and businesses in many countries. ADSL modems use a form of multicarrier modulation known as discrete multitone (DMT) modulation. DMT modulation provides an efficient means to combat channel dispersion. The efficiency arises from the use of the fast Fourier transform (FFT) to perform multicarrier modulation and simplify frequency domain equalization. ADSL+, ADSL2, and VDSL DMT standards have been approved over the last two years.

Multicarrier modulation partitions a broadband channel into a large number of approximately independent narrowband subchannels. With the assumption that each subchannel has a flat frequency response, the total number of bits transmitted over the entire bandwidth would be the sum of the bits transmitted in each subchannel. In DMT modulation, the number of bits assigned to each subchannel is determined by the receiver based on the signal-to-noise (SNR) ratio in that subchannel only (i.e., independent of that of other subchannels). This bit loading has the potential to maximize the achieved bit rate.

Multicarrier modulation uses a cyclic prefix $(\mathrm{CP})$ as a time guard band to nullify intersymbol interference (ISI) and intercarrier interference (ICI) if the channel memory is not longer than CP length. For many wireline systems, including ADSL, channel memory is generally longer than the CP length, which causes ISI and ICI. The ISI and ICI dra-

M. Ding carried out this research while a full time student at UT Austin. Both authors were supported by The State of Texas Advanced Technology Program under project 003658-0614-2001 matically lower the subchannel SNR, which in turn leads to significant degradation in achievable bit rate. Reliable channel equalization is necessary to combat the severe ISI and ICI.

A conventional DMT equalizer consists of a cascade of a time-domain equalizer (TEQ), a multicarrier demodulator (FFT), and a frequency-domain equalizer (FEQ). The TEQ is an finite impulse response (FIR) filter. The FIR filter, which is in cascade with the discretized channel, shortens the cascaded channel memory to be CP length or shorter. The FEQ compensates for the phase and amplitude distortion of the shortended channel in the FFT domain by a single division per subchannel.

Many TEQ design methods have been proposed to optimize different criteria based on a training sequence. In this paper, we focus on four TEQ design methods: minimum mean squared error (MMSE) [1], [2], maximum shortening SNR (MSSNR) [3], minimum intersymbol interference (Min-ISI) [4], [5], and minimum delay spread (MDS) [6]. These four TEQ design methods have competitive bit rate performance vs. implementation complexity.

Optimum TEQ design methods often assume perfect channel knowledge and either fully rely on this knowledge to train the TEQ or use the channel knowledge to develop a fast computation method. In practical implementations, it is necessary to estimate the channel from part or all of the training sequence before one can proceed to design the TEQ. However, it is not well known how the channel estimation error affects the bit rate performance. Also, there is no explicit expression of bit rate loss is given in literature for a DMT system with channel estimation error.

In this paper, we derive a closed form model to express the bit rate loss in a DMT system due to channel estimation error. This model is generated based on previous research results of unified approach of multicarrier equalization [7] and eigenvalue perturbation theory [8]. Through computer simulations, we compare the sensitivity of bit rate performance to the channel estimation errors of the four wellknown channel knowledge based equalization methods.

\section{BACKGROUND}

\section{A. Bit loading in DMT}

A DMT transmitter exploits an $N$-point Inverse FFT (IFFT) to create $N / 2$ orthogonal subchannels. For large $N$ and adequately long $\mathrm{CP}$, the channel gain and noise power 
in each subchannel are flat. SNR for the $i$ th subchannel is estimated as

$$
\mathrm{SNR}_{i}=\frac{E\left[\left|X_{i}\right|^{2}\right]}{E\left[\left|X_{i}-\hat{X}_{i}\right|^{2}\right]}
$$

where $E$ stands for expectation, $X_{i}$ and $\hat{X}_{i}$ s are the transmitted and received symbol on the $i$ th subchannel. Number of bits assigned to $i$ th subchannel is then determined by

$$
b_{i}=\log _{2}\left(1+\frac{S N R_{i}}{\Gamma}\right)
$$

where $\Gamma$ is SNR gap for achieving Shannon channel capacity and is constant over all subchannels given the same target bit error rate for all subchannels. Bit rate of the system is calculated as

$$
R=f_{s} * \sum_{i \in \mathcal{S}} b_{i}
$$

where $f_{s}$ is symbol rate and $\mathcal{S}$ is the set of all used subchannels.

\section{B. TEQ design}

The insertion of the CP of length $\nu$ samples enables the the linear convolution of a length $N$ DMT symbol and a channel impulse response up to length $\nu+1$ to be equal to a length $N$ circular convolution between them. This simplifies equalization in DFT domain on a per subchannel basis, but reduces the throughput of the channel. To minimize this reduction of throughput, a TEQ is applied to reduce the overall duration of the system (channel plus equalizer) impulse response to a predefined length.

Most published time domain equalizer design methods can be unified as a maximization of a generalized Rayleigh quotient, including MMSE, MSSNR, Min-ISI and MDS [7]:

$$
\mathbf{w}^{\text {opt }}=\arg \max _{\mathbf{w}} \frac{\mathbf{w}^{T} \mathbf{B} \mathbf{w}}{\mathbf{w}^{T} \mathbf{A} \mathbf{w}}
$$

Here, $\mathbf{w}$ is a $L_{w} \times 1$ vector containing the TEQ coefficients. $\mathbf{A}$ and $\mathbf{B}$ are generally different matrices when formulated under different criteria. The solution is the generalized eigenvector of the matrix pair $(\mathbf{B}, \mathbf{A})$ corresponding to the largest generalized eigenvalue.

\section{Bit Rate Loss Model}

\section{A. General Formulation}

The received signal $\hat{X}_{i}$ for the $i$ th subchannel at the FFT output can be written as

$$
\hat{X}_{i}=\phi_{i} \mathbf{q}_{i}^{H} \mathbf{Y} \mathbf{w}
$$

where $\mathbf{Y}$ is a $N \times L_{w}$ Toeplitz matrix which contains the received signal at channel output

$$
\mathbf{Y}=\left[\begin{array}{ccc}
y_{t}(\nu) & \cdots & y_{t}\left(\nu-L_{w}+1\right) \\
y_{t}(\nu+1) & \cdots & y_{t}\left(\nu-L_{w}+2\right) \\
\vdots & \ddots & \vdots \\
y_{t}(N+\nu-1) & \ldots & y_{t}\left(N+\nu-L_{w}\right)
\end{array}\right]
$$

$\mathbf{q}_{i}^{H}$ is the $i$ th row of DFT matrix and $\phi_{i}$ is the one tap $i$ th FEQ. Follow the approaches provided in [9], [10] by choosing an unbiased zero forcing FEQ, we could have

$$
\phi_{i}=\frac{E\left[\left|X_{i}\right|^{2}\right]}{E\left[\mathbf{q}_{i}^{H} \mathbf{Y} X_{i}^{*}\right] \mathbf{w}}
$$

Substitute (5) and (7) into (1), and after some manipulations, we have

$$
\mathrm{SNR}_{i}=\frac{\left|E\left[\mathbf{q}_{i}^{H} \mathbf{Y} X_{i}^{*}\right] \mathbf{w}\right|^{2}}{\mathbf{w}^{T} E\left[\mathbf{Y}^{H} \mathbf{q}_{i} \mathbf{q}_{i}^{H} \mathbf{Y}\right] \mathbf{w}-\left|E\left[\mathbf{q}_{i}^{H} \mathbf{Y} X_{i}^{*}\right] \mathbf{w}\right|^{2}}
$$

With this SNR model, the bit rate is actually a nonlinear function of the TEQ coefficients:

$$
R=f_{s} * \sum_{i \in \mathcal{S}} \log _{2}\left(\frac{\mathbf{w}^{T} \mathbf{V}_{i} \mathbf{w}}{\mathbf{w}^{T} \mathbf{U}_{i} \mathbf{w}}\right)
$$

where

$$
\begin{aligned}
\mathbf{V}_{i}= & \Gamma E\left[\left|X_{i}\right|^{2}\right] E\left[\mathbf{Y}^{H} \mathbf{q}_{i} \mathbf{q}_{i}^{H} \mathbf{Y}\right] \\
& +(1-\Gamma) E\left[\mathbf{Y}^{H} \mathbf{q}_{i} X_{i}\right] E\left[X_{i}^{*} \mathbf{q}_{i}^{H} \mathbf{Y}\right] \\
\mathbf{U}_{i}= & \Gamma\left(E\left[\left|X_{i}\right|^{2}\right] E\left[\mathbf{Y}^{H} \mathbf{q}_{i} \mathbf{q}_{i}^{H} \mathbf{Y}\right]\right. \\
& \left.-E\left[\mathbf{Y}^{H} \mathbf{q}_{i} X_{i}\right] E\left[X_{i}^{*} \mathbf{q}_{i}^{H} \mathbf{Y}\right]\right)
\end{aligned}
$$

For the optimum design with perfect channel knowledge, we calculate bit rate by substituting (4) into (9). However, the TEQ training usually ends up at a non-optimum $\tilde{\mathbf{w}}$ due to the presence of channel estimation error. We assume in (4), $\mathbf{A}$ and $\mathbf{B}$ are replaced by $\mathbf{A}+\Delta \mathbf{A}$ and $\mathbf{B}+\Delta \mathbf{B}$. Due to the different formulations of $\mathbf{A}$ and $\mathbf{B}$ in various channel estimation based methods, $\Delta \mathbf{A}$ and $\Delta \mathbf{B}$ are not the same error matrix in general. The generalized eigen-problem of TEQ design can be reduced to finding an eigenvector of $\mathbf{C}=\mathbf{A}^{-1} \mathbf{B}$. Though in practice we consider other approaches to solve it due to numerical implementation concerns, this approach could serve here as an analytic study of channel estimation error effects.

Suppose a $n \times n$ matrix $\mathbf{C}$, which has $n$ eigenvalues $\lambda_{i} \mathrm{~s}$, $n$ corresponding eigenvectors $\mathbf{w}_{i} \mathrm{~s}$ and $n$ left eigenvectors $\mathbf{p}_{i}$ s, is perturbed by $\tilde{\mathbf{C}}=\mathbf{C}+\Delta \mathbf{C}$. We have

$$
\begin{aligned}
\tilde{\mathbf{C}}= & (\mathbf{A}+\Delta \mathbf{A})^{-1}(\mathbf{B}+\Delta \mathbf{B}) \\
= & \left(\mathbf{A}^{-1}-\mathbf{A}^{-1}\left(\Delta \mathbf{A}^{-1}+\mathbf{A}^{-1}\right)^{-1} \mathbf{A}^{-1}\right)(\mathbf{B}+\Delta \mathbf{B}) \\
= & \mathbf{A}^{-1} \mathbf{B}-\mathbf{A}^{-1}\left(\Delta \mathbf{A}^{-1}+\mathbf{A}^{-1}\right)^{-1} \mathbf{A}^{-1} \mathbf{B} \\
& \quad+\mathbf{A}^{-1} \Delta \mathbf{B}-\mathbf{A}^{-1}\left(\Delta \mathbf{A}^{-1}+\mathbf{A}^{-1}\right)^{-1} \mathbf{A}^{-1} \Delta \mathbf{B} \\
= & \mathbf{C}+\Delta \mathbf{C}
\end{aligned}
$$

where $\Delta \mathbf{C}$ is the sum of last three items in (11)

In a practical TEQ design, usually $\mathbf{C}$ has only one largest eigenvalue $\lambda_{k}$, and optimum TEQ $\mathbf{w}=\mathbf{w}_{k}$ in this case. Under the perturbation of $\Delta \mathbf{C}, \lambda_{k}$ is replaced by $\lambda_{k}+\Delta \lambda_{k}$ and $\mathbf{w}_{k}$ is changed to $\mathbf{w}_{k}+\Delta \mathbf{w}_{k}$. Write

$$
\Delta \mathbf{w}_{k}=\sum_{i=1}^{n} d_{i} \mathbf{w}_{i}
$$


where $d_{i}$ are projection coefficients. We have

$$
\mathbf{w}_{k}+\Delta \mathbf{w}_{k}=\left(1+d_{k}\right) \mathbf{w}_{k}+\sum_{i \neq k} d_{i} \mathbf{w}_{i}
$$

Since eigenvectors are determined only up to a scalar multiple, we can always set $d_{k}=0$ to make $\Delta \mathbf{w}_{k}=\sum_{i \neq k} d_{i} \mathbf{w}_{i}$. We follow the approach in [8] to expand

$$
(\mathbf{C}+\Delta \mathbf{C})\left(\mathbf{w}_{k}+\Delta \mathbf{w}_{k}\right)=\left(\lambda_{k}+\Delta \lambda_{k}\right)\left(\mathbf{w}_{k}+\Delta \mathbf{w}_{k}\right) .
$$

Using the facts

$$
\begin{aligned}
\mathbf{C} \mathbf{w}_{i} & =\lambda_{i} \mathbf{w}_{i} \\
\mathbf{p}_{j}^{H} \mathbf{w}_{k} & =0 \text { if } k \neq j \text { and } \mathbf{p}_{k}^{H} \mathbf{w}_{k} \neq 0,
\end{aligned}
$$

it can be shown that

$$
\begin{aligned}
\Delta \mathbf{w}_{k} & =\alpha^{-1} \sum_{k \neq i} \mathbf{w}_{i} \frac{\mathbf{p}_{i}^{H}(\Delta \mathbf{C}) \mathbf{w}}{\left(\lambda_{k}-\lambda_{i}\right) \mathbf{p}_{i}^{H} \mathbf{w}_{i}} \\
\tilde{\mathbf{w}} & =\mathbf{w}+\Delta \mathbf{w}_{k}=\beta \mathbf{w}
\end{aligned}
$$

where

$$
\begin{aligned}
\alpha=\mathbf{I} & +\sum_{k \neq i} \mathbf{w}_{i} \frac{\mathbf{p}_{i}^{H}\left(\Delta \lambda_{k}\right)}{\left(\lambda_{k}-\lambda_{i}\right) \mathbf{p}_{i}^{H} \mathbf{w}_{i}} \\
& -\sum_{k \neq i} \mathbf{w}_{i} \frac{\mathbf{p}_{i}^{H}(\Delta \mathbf{C})}{\left(\lambda_{k}-\lambda_{i}\right) \mathbf{p}_{i}^{H} \mathbf{w}_{i}}, \\
\beta= & \mathbf{I}+\alpha^{-1} \sum_{k \neq i} \mathbf{w}_{i} \frac{\mathbf{p}_{i}^{H}(\Delta \mathbf{C})}{\left(\lambda_{k}-\lambda_{i}\right) \mathbf{p}_{i}^{H} \mathbf{w}_{i}},
\end{aligned}
$$

and $\mathbf{I}$ is $L_{w} \times L_{w}$ identity matrix. Hence, the bit rate

$$
\begin{aligned}
\tilde{R} & =f_{s} * \sum_{i \in \mathcal{S}} \log _{2}\left(\frac{\tilde{\mathbf{w}}^{T} \mathbf{V}_{i} \tilde{\mathbf{w}}}{\tilde{\mathbf{w}}^{T} \mathbf{U}_{i} \tilde{\mathbf{w}}}\right) \\
& =f_{s} \log _{2} \prod_{i \in \mathcal{S}}\left(\frac{\tilde{\mathbf{w}}^{T} \mathbf{V}_{i} \tilde{\mathbf{w}}}{\tilde{\mathbf{w}}^{T} \mathbf{U}_{i} \tilde{\mathbf{w}}}\right)
\end{aligned}
$$

Data rate loss due to imperfect channel estimation can be written as

$$
\begin{aligned}
\Delta R & =f_{s}\left(\log _{2} \prod_{i \in \mathcal{S}}\left(\frac{\mathbf{w}^{T} \mathbf{V}_{i} \mathbf{w}}{\mathbf{w}^{T} \mathbf{U}_{i} \mathbf{w}}\right)-\log _{2} \prod_{i \in \mathcal{S}}\left(\frac{\tilde{\mathbf{w}}^{T} \mathbf{V}_{i} \tilde{\mathbf{w}}}{\tilde{\mathbf{w}}^{T} \mathbf{U}_{i} \tilde{\mathbf{w}}}\right)\right) \\
& =f_{s} \log _{2} \prod_{i \in \mathcal{S}}\left(\frac{\mathbf{w}^{T} \mathbf{V}_{i} \mathbf{w}(\beta \mathbf{w})^{T} \mathbf{U}_{i}(\beta \mathbf{w})}{\mathbf{w}^{T} \mathbf{U}_{i} \mathbf{w}(\beta \mathbf{w})^{T} \mathbf{V}_{i}(\beta \mathbf{w})}\right)
\end{aligned}
$$

\section{B. Case Studies}

With the unified approach proposed above, we look into each different design to find out what is $\Delta \mathbf{C}$ in each case.

\section{B.1 Maximum Shortening SNR}

The MSSNR approach [3] is based solely on shortening the channel impulse response. We define channel convolution matrix as,

$$
\mathbf{H}=\left[\begin{array}{cccc}
h(0) & h(-1) & \ldots & h\left(-\left(L_{w}-1\right)\right) \\
h(1) & h(0) & \ldots & h\left(-\left(L_{w}-2\right)\right) \\
\vdots & \vdots & \ddots & \vdots \\
h(N-1) & h(N-2) & \ldots & h\left(N-L_{w}\right)
\end{array}\right]
$$

and a sliding shortening window function as

$$
g(n)= \begin{cases}1 & \text { if } \Delta \leq n \leq \Delta+\nu \\ 0 & \text { elsewhere }\end{cases}
$$

where $\Delta$ is the transmission delay. Further, we defined

$$
\mathbf{G}=\operatorname{diag}[g(0) \quad g(1) \quad \ldots \quad g(N-1)]^{T}
$$

and $\mathbf{D}=\mathbf{I}-\mathbf{G}$, we have

$$
\begin{aligned}
& \mathbf{A}=\mathbf{H}^{T} \mathbf{D}^{T} \mathbf{D H} \\
& \mathbf{B}=\mathbf{H}^{T} \mathbf{G}^{T} \mathbf{G} \mathbf{H}
\end{aligned}
$$

Channel estimation error is defined as perturbation to matrix $\mathbf{H}$ as $\Delta \mathbf{H}$. Assume $\|\Delta \mathbf{H}\|_{2}=\epsilon$ is sufficiently small, we have

$$
\begin{aligned}
\tilde{\mathbf{A}} & =(\mathbf{H}+\Delta \mathbf{H})^{T} \mathbf{D}^{T} \mathbf{D}(\mathbf{H}+\Delta \mathbf{H})=\mathbf{A}+\Delta \mathbf{A} \\
\tilde{\mathbf{B}} & =(\mathbf{H}+\Delta \mathbf{H})^{T} \mathbf{G}^{T} \mathbf{G}(\mathbf{H}+\Delta \mathbf{H})=\mathbf{B}+\Delta \mathbf{B}
\end{aligned}
$$

where

$$
\begin{aligned}
\Delta \mathbf{A} & =\mathbf{H}^{T} \mathbf{D}^{T} \mathbf{D} \Delta \mathbf{H}+\Delta \mathbf{H}^{T} \mathbf{D}^{T} \mathbf{D} \mathbf{H}+\mathcal{O}\left(\epsilon^{2}\right) \\
\Delta \mathbf{B} & =\mathbf{H}^{T} \mathbf{G}^{T} \mathbf{G} \Delta \mathbf{H}+\Delta \mathbf{H}^{T} \mathbf{G}^{T} \mathbf{G} \mathbf{H}+\mathcal{O}\left(\epsilon^{2}\right)
\end{aligned}
$$

$\Delta \mathbf{C}$ is then easily computed from $\mathbf{A}, \mathbf{B}, \Delta \mathbf{A}$, and $\Delta \mathbf{B}$. TEQ and bit rate loss computation can proceed straight forwardly.

\section{B.2 Min-ISI}

The Min-ISI method generalizes the MSSNR method by weighting the ISI in the frequency domain [4], [5], e.g., to place the ISI in unused and low SNR subchannels. Similarly, for Min-ISI, we have

$$
\begin{aligned}
\Delta \mathbf{A}= & \mathbf{H}^{T} \mathbf{D}^{T}\left(\sum_{i \in \mathcal{S}} \mathbf{q}_{i}^{H} \frac{S_{x, i}}{S_{n, i}} \mathbf{q}_{i}\right) \mathbf{D} \Delta \mathbf{H} \\
& +\Delta \mathbf{H}^{T} \mathbf{D}^{T}\left(\sum_{i \in \mathcal{S}} \mathbf{q}_{i}^{H} \frac{S_{x, i}}{S_{n, i}} \mathbf{q}_{i}\right) \mathbf{D H}+\mathcal{O}\left(\epsilon^{2}\right) \\
\Delta \mathbf{B}= & \mathbf{H}^{T} \mathbf{G}^{T} \mathbf{G} \Delta \mathbf{H}+\Delta \mathbf{H}^{T} \mathbf{G}^{T} \mathbf{G} \mathbf{H}+\mathcal{O}\left(\epsilon^{2}\right)
\end{aligned}
$$

\section{B.3 MDS}

MDS method [6] is to minimize so called delay spread of the effective channel impulse response. Delay spread is defined as

$$
D=\sqrt{\frac{1}{h_{e}} \sum_{n=0}^{L_{h}}(n-\bar{n})^{2}|h(n)|^{2}}
$$

where $h_{e}$ is the energy of channel impulse response, and $\bar{n}$ is a user-defined center tap of $h$.

$$
\begin{aligned}
\Delta \mathbf{A} & =\mathbf{H}^{T} \mathbf{Q} \Delta \mathbf{H}+\Delta \mathbf{H}^{T} \mathbf{Q H}+\mathcal{O}\left(\epsilon^{2}\right) \\
\Delta \mathbf{B} & =\mathbf{H}^{T} \Delta \mathbf{H}+\Delta \mathbf{H}^{T} \mathbf{H}+\mathcal{O}\left(\epsilon^{2}\right)
\end{aligned}
$$

where $\mathbf{Q}=\operatorname{diag}\left\{\left[(0-\bar{n})^{2}, \ldots\left(L_{w}+L_{h}-\bar{n}\right)^{2}\right]\right\}$ is a diagonal weighting matrix. 


\section{B.4 MMSE}

MMSE TEQ design [1], [2] minimizes the mean square error between the output of the physical path consisting of the channel and FIR filter and the output of a virtual path consisting of a transmission delay $\Delta$ and a target impulse response (TIR). In the case of MMSE TIR with unit norm constraint, the solution to the generalized eigenvalue problem is the optimum target impulse response $\mathbf{b}$.

$$
\begin{aligned}
\mathbf{A} & =\left(\Psi^{T} \mathbf{R}_{x} \Psi\right)-\Psi^{T} \mathbf{R}_{x} \mathbf{H}\left(\mathbf{H}^{T} \mathbf{R}_{x} \mathbf{H}+\mathbf{R}_{n}\right)^{-1} \mathbf{H}^{T} \mathbf{R}_{x} \Psi \\
& =\left[\left(\Psi^{T} \mathbf{R}_{x} \Psi\right)^{-1}+\mathbf{H R}_{n}^{-1} \mathbf{H}^{T}\right]^{-1} \\
\mathbf{B} & =\mathbf{I}_{\nu+1}
\end{aligned}
$$

where $\Psi$ is a $\left(L_{h}+L_{w}-1\right) \times(\nu+1)$ windowing matrix defined as

$$
[\Psi]_{m, n}=\delta(m+n-\Delta)\left\{\begin{array}{l}
0 \leq m<L_{w}+L_{h}-1 \\
0 \leq n<\nu+1
\end{array}\right.
$$

Directly compute $\Delta \mathbf{C}$ is easier in this case,

$$
\begin{aligned}
\tilde{\mathbf{C}} & =\left(\Psi^{T} \mathbf{R}_{x} \Psi\right)^{-1}+(\mathbf{H}+\Delta \mathbf{H}) \mathbf{R}_{n}^{-1}(\mathbf{H}+\Delta \mathbf{H})^{T} \\
\Delta \mathbf{C} & =\mathbf{H R}_{n}^{-1} \Delta \mathbf{H}^{T}+\Delta \mathbf{H} \mathbf{R}_{n}^{-1} \mathbf{H}^{T}+\mathcal{O}\left(\epsilon^{2}\right)
\end{aligned}
$$

Once we obtain $\tilde{\mathbf{b}}=\beta \mathbf{b}$, the TEQ $\tilde{\mathbf{w}}$ can be calculated

$$
\begin{aligned}
\tilde{\mathbf{w}}= & \left((\mathbf{H}+\Delta \mathbf{H})^{T} \mathbf{R}_{x}(\mathbf{H}+\Delta \mathbf{H})+\mathbf{R}_{n}\right)^{-1} \\
& (\mathbf{H}+\Delta \mathbf{H})^{T} \mathbf{R}_{x} \Psi \tilde{\mathbf{b}}
\end{aligned}
$$

\section{Simulations}

The simulations compare the sensitivity to bit rate performance of the different equalizer designs for a wireline communication transceiver. More specifically, we consider a downstream first generation ADSL transmission. According to the ITU ADSL standard, the IFFT and FFT lengths are 512 and the cyclic prefix length is 32 . We test our designs on eight typical carrier service area (CSA) loops recommended by Bell Labs [11]. Full ADSL bandwidth is up to $1.104 \mathrm{MHz}$. A common practice in industry is to use frequency division multiplexing to allocate bi-directional transmission to different frequency bands. We adopt this approach and introduce a 5th order high pass IIR filter with passband frequency at $138 \mathrm{kHz}$ to separate the downstream data from the upstream data. The signal power spectral density at the transmitter output is set equal to $-40 \mathrm{dBm} / \mathrm{Hz}$. Channel noise is modeled as an additive white Gaussian noise (AWGN) with $-140 \mathrm{dBm} / \mathrm{Hz}$ power density, NEXT noise from 5 integrated services digital network (ISDN) disturbers.

Fig. 1 presents magnitude responses of the eight test loops. The average channel impulse response power is between $-43 \mathrm{dBm}$ and $-48 \mathrm{dBm}$. In our system setup, the average received signal power $P_{r}$ is around $-24 \mathrm{dBm}$ and noise power $P_{n}$ (including crosstalk and AWGN) is about $60 \mathrm{dBm}$. We model channel estimation error as an AWGN noise with variance $\sigma^{2}$. According to [12], if we adopt a commonly used frequency domain channel estimates

$$
\hat{H}_{i}=\frac{1}{L} \sum_{k=1}^{L} \frac{R_{k, i}}{X_{i}}
$$

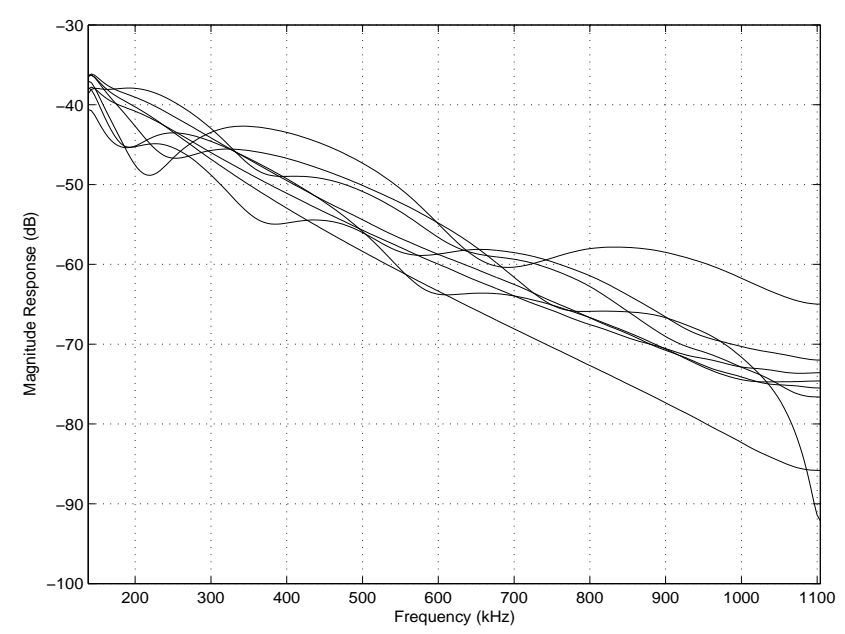

Fig. 1. Magnitude responses in downstream transmission bandwidth for eight CSA loops

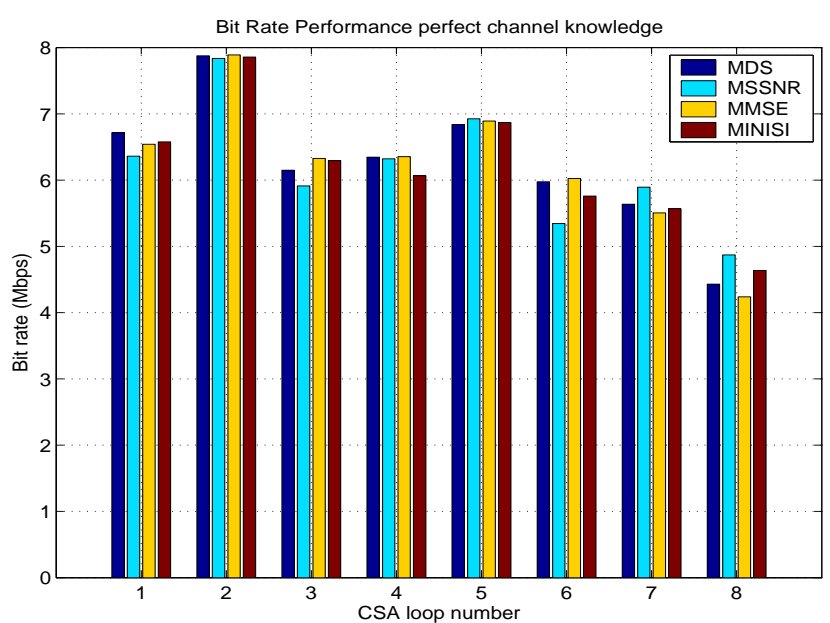

Fig. 2. Achievable bit rate for $8 \mathrm{CSA}$ loops with perfect channel knowledge Coding gain is $5 \mathrm{~dB}$, margin is $6 \mathrm{~dB}$, input power is $-40 \mathrm{dBm} / \mathrm{Hz}$, AWGN power $-140 \mathrm{dBm} / \mathrm{Hz}, \mathrm{NEXT}$ noise is from 5 ISDN disturbers. Equalizer is trained by the MDS, MSSNR, MMSE and Min-ISI.

where $R_{k, i}$ is the $i$ th DFT element of received channel output at $k$ th cycle, the channel estimation error is controlled by $\sigma^{2}=\frac{1}{L} P_{n}$. We choose a reasonable $\sigma^{2}$ ranging from -90 $\mathrm{dBm}$ to $-76 \mathrm{dBm}$, where end points corresponding to averaging on $L=1000$ cycles and $L=40$ cycles, respectively. $L=40$ is also suggested in [12] as a lower bound of estimation cycles. Moreover, our channel estimation error power is corresponding to an AWGN with power spectral density from $-153 \mathrm{dBm} / \mathrm{Hz}$ to $-133 \mathrm{dBm} / \mathrm{Hz}$ within our transmission bandwidth, which is significantly below channel gain in this range. It further suggests our choice of estimation error power is fairly conservative.

The SNR gap to Shannon capacity in our simulation is chosen as

$\Gamma_{\text {sim }}($ in $\mathrm{dB})=\Gamma_{\text {gap }}+$ system margin - coding gain $(32)$

where $\Gamma_{\text {gap }}=9.8 \mathrm{~dB}$ corresponds to $10^{-7}$ bit error rate, 


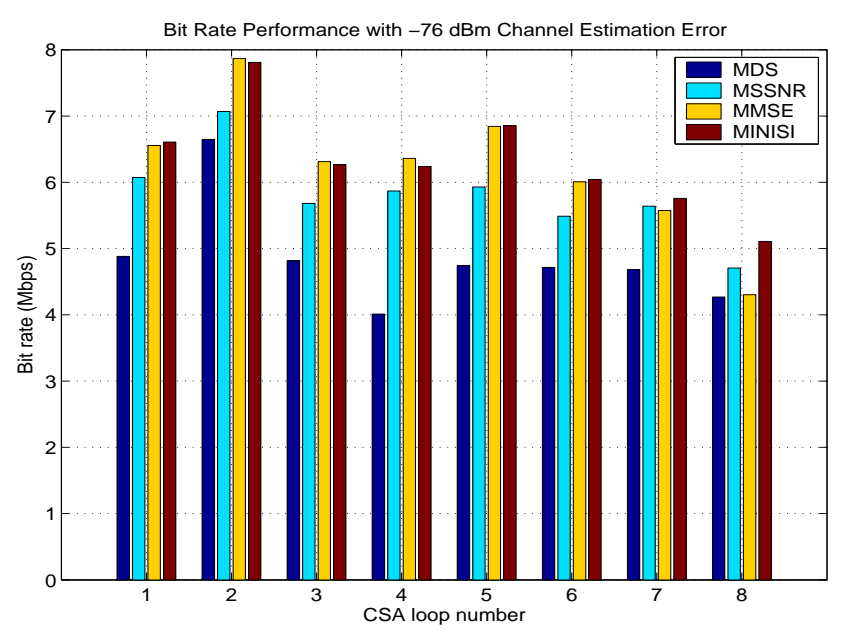

Fig. 3. Achievable bit rate for 8 CSA loops with $-76 \mathrm{dBm}$ channel estimation error Coding gain is $5 \mathrm{~dB}$, margin is $6 \mathrm{~dB}$, input power is $-40 \mathrm{dBm} / \mathrm{Hz}$, AWGN power $-140 \mathrm{dBm} / \mathrm{Hz}, \mathrm{NEXT}$ noise is from 5 ISDN disturbers. Equalizer is trained by the MDS, MSSNR, MMSE and Min-ISI.

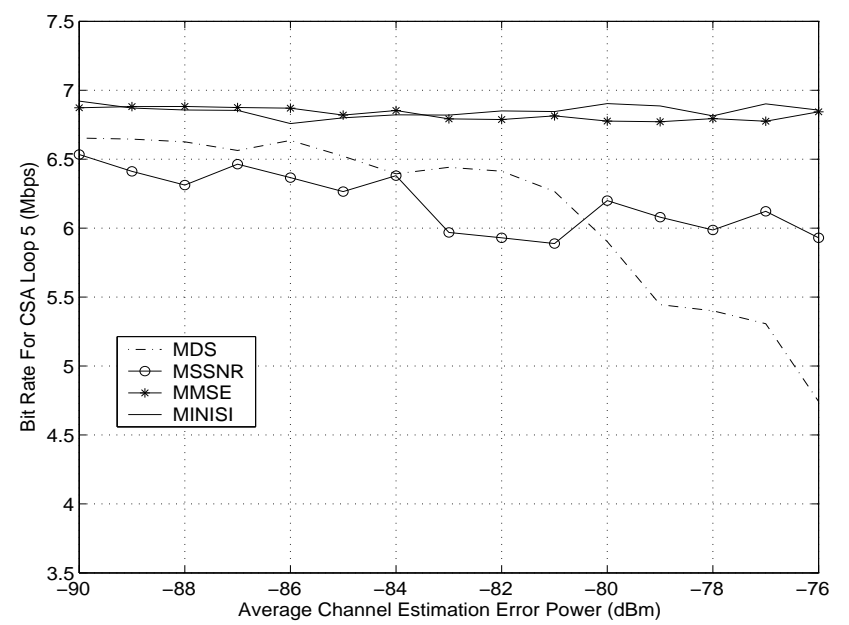

Fig. 4. Achievable bit rate for CSA loop 5 with channel estimation error from $-90 \mathrm{dBm}$ to $-76 \mathrm{dBm}$. Coding gain is $5 \mathrm{~dB}$, margin is 6 $\mathrm{dB}$, input power is $-40 \mathrm{dBm} / \mathrm{Hz}$, AWGN power $-140 \mathrm{dBm} / \mathrm{Hz}$, NEXT noise is from 5 ISDN disturbers. Equalizer is trained by the MDS, MSSNR, MMSE and Min-ISI.

system margin is $6 \mathrm{~dB}$, and coding gain is $5 \mathrm{~dB}$.

Fig. 2 displays the achievable bit rates for eight CSA loops with perfect channel estimation. Though the four design methods use different metric to optimize TEQ settings, the bit rate performances are quite close with full knowledge of channel impulse response.

Fig. 3 displays the achievable bit rates for eight CSA loops when $-76 \mathrm{dBm}$ power channel estimation error is introduced. It appears that Min-ISI and MMSE outperform MSSNR by roughly $10 \%$ and MDS by roughly $20 \%$. The performance gap is universally perceivable among all eight loops. It suggests the Min-ISI and MMSE performance hold better against channel estimation error than the performance of MSSNR and MDS.
Fig. 4 shows bit rate vs. channel estimation error power for loop 5. In Fig. 4, MSSNR and MDS are significantly affected by channel estimation error. MSSNR and MDS completely depend on the channel impulse response. In addition, MDS uses a $\mathbf{Q}$ weighting matrix to amplify the impulse response as well as the estimation error. On the other hand, Min-ISI and MMSE have already taken noise into account, and hence, are relatively insensitive to channel estimation error. With accurate channel gain estimates, estimation error power can be $16 \mathrm{~dB}$ lower than additive noise power, which would likely not affect the bit rate performance as much as observed in simulations.

Since none of the four methods directly optimize the bit rate function in (2), a TEQ design with small estimation error could achieve a higher bit rate in some cases. This means $\Delta R$ of (21) might be negative in some cases.

\section{Conclusions}

This paper studies the behavior of bit rate performance of four popular TEQ designs when channel estimation error is present. A rate loss model as a function of optimum TEQ with perfect channel knowledge is provided. Through extensive simulations with practical noise injection, we conclude that the bit rate performance of Min-ISI and MMSE are robust against imperfect channel knowledge, while the performance of MSSNR and MDS are relatively sensitive to channel estimation error.

\section{REFERENCES}

[1] J. S. Chow, J. M. Cioffi, and J. A. C. Bingham, "Equalizer Training Algorithms for Multicarrier Modulation Systems," in Proc. IEEE Int. Conf. on Comm., May 1993, pp. 761-765.

[2] N. Al-Dhahir and J. M. Cioffi, "Efficiently Computed ReducedParameter Input-Aided MMSE Equalizers for ML Detection: A Unified Approach," IEEE Trans. on Info. Theory, vol. 42, no. 3, pp. 903-915, May 1996.

[3] P. J. W. Melsa, R. C. Younce, and C. E. Rohrs, "Impulse Response Shortening for Discrete Multitone Transceivers," IEEE Trans. on Comm., vol. 44, pp. 1662-1672, Dec. 1996.

[4] G. Arslan, B. L. Evans, and S. Kiaei, "Equalization for Discrete Multitone Receivers To Maximize Bit Rate," IEEE Trans. on Sig. Proc., vol. 49, no. 12, pp. 3123-3135, Dec. 2001.

[5] M. Ding, B. L. Evans, R. K. Martin, and C. R. Johnson, "Minimum Intersymbol Interference Methods for Time Domain Equalizer Design," in Proc. IEEE Global Comm. Conf., Dec. 2003, vol. 4, pp. $2146-2150$.

[6] R. Schur and J. Speidel, "An Efficient Equalization Method to Minimize Delay Spread in OFDM/DMT Systems," in Proc. IEEE Int. Conf. on Comm., June 2001.

[7] R. K. Martin, K. Vanbleu, M. Ding, G. Ysebaert, M. Milosevic, B. L. Evans, M. Moonen, and C. R. Johnson, "Unification and Evaluation of Equalization Structures and Design Algorithms for Discrete Multitone Modulation Systems," to appear in IEEE Trans. on Sig. Proc., 2005.

[8] J. Demmel, Applied Numerical Linear Algebra, SIAM, 1997.

[9] K. Vanbleu, G. Ysebaert, G. Cuypers, M. Moonen, and K. Van Acker, "Bitrate Maximizing Time-Domain Equalizer Design for DMT-based Systems," in Proc. IEEE Int. Conf. on Comm., May 2003, pp. 2360-2364.

[10] R. K. Martin, K. Vanbleu, M. Ding, G. Ysebaert, M. Milosevic, B. L. Evans, M. Moonen, and C. R. Johnson, "Implementation Complexity and Communication Performance Tradeoffs in Discrete Multitone Modulation Equalizers," in preparation.

[11] G. Arslan, M. Ding, B. Lu, M. Milosevic, Z. Shen, and B. L. Evans, "DMTTEQ design toolbox," The University of Texas at Austin, www.ece.utexas.edu/ bevans/projects/adsl/dmtteq/.

[12] J. M. Cioffi, EE379C Course Notes, Stanford University, 2001. 